\title{
The effect of controlled microrobotized blasting on implant surface texturing and early osseointegration
}

\author{
Luiz F Gil', Charles Marin ${ }^{2}$, Hellen Teixeira ${ }^{3}$, Heloisa F Marão ${ }^{4}$, \\ Nick Tovar $^{5}$, Rehan Khan ${ }^{5}$, Estevam A Bonfante ${ }^{6}$, Malvin Janal ${ }^{7}$ \\ and Paulo G Coelho $3,5,8,9$
}

\begin{abstract}
Surface topography modifications have become a key strategy for hastening the host-to-implant response to implantable materials. The present study evaluated the effect of three different carefully controlled surface texture patterns achieved through microrobotized blasting (controlled to high, medium and low roughness) relative to a larger scale blasting procedure (control) in early osseointegration in a canine model. Four commercially pure grade 2 titanium alloy implants (one of each surface) were bilaterally placed in the radii of six beagle dogs and allowed end points of I and 6 weeks in vivo. Following sacrifice, implants in bone were non-decalcified processed for bone morphologic and histometric (bone-to-implant contact; bone area fraction occupancy) evaluation. Surface topography was characterized by scanning electron microscopy and optical interferometry. Results showed initial osteogenic tissue interaction at one week and new bone in intimate contact with all implant surfaces at 6 weeks. At I and 6 weeks in vivo, higher bone-to-implant and bone area fraction occupancy were observed for the high texture pattern microrobotized blasted surface relative to others.
\end{abstract}

\section{Keywords}

Implant surface, surface roughness, bone, osseointegration, histology

\section{Introduction}

Over the course of five decades, the phenomenon of osseointegration has gone from discovery to commodity for a variety of rehabilitative procedures in dentistry and medicine. ${ }^{1}$ The success of this ankylotic phenomenon is dependent on numerous factors ${ }^{2}$ that include implant design, ${ }^{3-5}$ device initial stability, ${ }^{3,4,6}$ adequate and minimally traumatic surgical procedure, ${ }^{6,7}$ bulk and surface biocompatiblity and osseoconductivity of devices, ${ }^{8-11}$ and the interplay between them. ${ }^{12}$

Among the design features of endosteal implants, surface topographical modification at the micro and nanometer length scales have by far been the most investigated, since it has the potential of initially change early biological cascades that influence the achievement of osseointegration. A plethora of surface modifications reported in the literature have shown potential to accelerate early bone healing around implants ${ }^{13-16}$ and to lead to earlier biomechanical stability competence of the bone-implant interface. ${ }^{17,18}$
'Universidade Federal de Santa Catarina, Department of Dentistry, Florianopolis, Brazil

${ }^{2}$ UNIGRANRIO University, Postgraduate Program in Dentistry, Duque de Caxias, Brazil

${ }^{3}$ University of Pennsylvania, School of Dental Medicine, Department of Orthodontics, Philadelphia, PA, USA (for Dr. Hellen Teixeira)

${ }^{4}$ São Paulo State University, Department of Surgery and Integrated Clinics, Araçatuba, Brazil

${ }^{5} \mathrm{New}$ York University, Department of Biomaterials and Biomimetics, New York, USA

${ }^{6}$ University of Sao Paulo - Bauru College of Dentistry, Department of Prosthodontics, Bauru, SP, Brazil (for Estevam A. Bonfante)

${ }^{7}$ Department of Epidemiology and Health Promotion, New York University College of Dentistry (for Dr. Malvin Janal)

${ }^{8}$ Director for Implant Research, Department of Periodontology and Implant Dentistry, New York University, New York, NY, USA (For Dr. Paulo G. Coelho)

${ }^{9}$ Affiliated Faculty, Department of Engineering, New York University Abu Dhabi, Abu Dhabi, United Arab Emirates. (For Dr. Paulo G. Coelho)

Corresponding author:

Estevam A Bonfante, University of São Paulo - Bauru College of Dentistry, Department of Prosthodontics, Al Octávio Pinheiro Brisola 975, Bauru 1701290I, Brazil.

Email: estevamab@gmail.com 
Surface texturing is a commonly applied surface modification method and has unequivocally shown to positively modulate the initial interaction between the implant surface and host biofluids. ${ }^{18}$ For instance, relative to the turned (machined) implant surfaces, micrometer scale texturing in the moderately roughened range has enabled earlier and improved degrees of biomechanical competence and bone-to-implant contact (BIC). ${ }^{18-20}$ One reason for such enhancement of osseointegration has been suggested to be an outcome of the intimate interaction between the modified implant surface and the surrounding tissue, which is initiated by the high affinity to attract blood clot and osteogenic proteins to its surface. ${ }^{21,22}$ Such tissue level interaction gain at the micrometer scale has been subsequently demonstrated to be of significant importance, especially for strategies that include nanometer scale texturing patterns. ${ }^{23}$ It has been suggested that nanometer scale surface patterns have some influence on the osteogenic cell phenotype expression via extracellular signaling pathways, which could have a significant impact on the advancement of tissue engineering. ${ }^{24}$ While it is general consensus that cell signaling is more efficiently achieved through nanometer scale design, it has been stressed that hierarchical topographical feature with a microtexturing is essential for osseointegration. ${ }^{12,25}$ Reportedly, the absence of a micrometer scale pattern on the surface of endosteal implants that carry nanometer scale features lead to less than optimal bone healing. ${ }^{12}$

From a manufacturing point of view, it is a great challenge to obtain controlled surface pattern designing that can increase or decrease the roughness patterns. This is one of the reasons why experimental studies that evaluate bone to implant response as a function of variations in surface patterning have not been conducted as much as the other study models. Such challenge has recently been minimized through the development of microrobotized blasting procedures that through the combination of blasting media type and size, blasting velocity, and surface coverage allow more precise control to surface roughness parameters. ${ }^{26}$ It has been described by Valverde et al. that through the combination of three different variables, the controlled development of 36 different surface patterns comprising surface texturing profiles that encompass the vast majority of commercially available surfaces was possible. ${ }^{26}$ Such microrobotized blasting procedure was shown to result in more uniform surface texturing than the often utilized manual or larger scale automated blasting procedures often employed for surface engineering by endosteal implant manufacturers.

In this study, we have tested in vivo three different surface texture profiles obtained through the controlled microrobotized blasting presented in our previous study. ${ }^{26}$ The selected surfaces included the two ends of the spectrum obtained (highest and lowest degrees of surface roughness) as well as an intermediate surface roughness pattern. For comparative purposes, a surface obtained through a larger scale blasting procedure was included.

\section{Materials and methods}

Forty-eight, commercially pure grade 2 titanium alloy, threaded endosseous implants, $4 \mathrm{~mm}$ in diameter and $10 \mathrm{~mm}$ in length (Novo Colosso, Emfils, Itu, Brazil), with a machined surface were provided by the manufacturer for microrobotized blasting procedures. A total of 16 implants presenting the same macrogeometric characteristics and with a surface treatment achieved through a larger scale blasting procedure were provided by the manufacturer as controls.

The three different microrobotized blasting procedures were carried out to provide low (L), medium (M), and high $(\mathrm{H})$ surface roughness profiles pre-established in our previous surface fabrication study. ${ }^{26}$ Control (C) implants were utilized as provided by the manufacturer. All experimental surfaces were blasted with a microrobotized device (Comco Inc, CA, USA) at a velocity of $150 \mathrm{~m} / \mathrm{s}$ and coverage of $5 \mathrm{~g} / \mathrm{in}^{2}$ and variations in particle size from $50 \mu \mathrm{m}$ to $100 \mu \mathrm{m}$ to $150 \mu \mathrm{m}$ resulted in the $\mathrm{L}, \mathrm{H}$, and $\mathrm{M}$ experimental surfaces, respectively. According to the manufacturer, the control surface was manually blasted with $50 \mu \mathrm{m}$ alumina particles at $\sim 45$ psi.

\section{Surface texture characterization}

For surface texture characterization, four implants per surface condition were utilized. Scanning electron microscopy (SEM, Philips XL 30, Eindhoven, The Netherlands) was performed at various magnifications under an acceleration voltage of $15 \mathrm{kV}$. Surface roughness was evaluated in the different implant surfaces by optical interferometry (IFM, Phase View 2.5, Palaiseau, France) at the flat region of the implant cutting edges (three measurements per implant). Sa (arithmetic average high deviation), $\mathrm{Sq}$ (root mean square), and $\mathrm{Sdr}$ (developed surface ratio) parameters were determined. A filter size of $250 \mu \mathrm{m} \times 250 \mu \mathrm{m}$ was utilized for a total of 15 measurements per surface.

\section{In vivo laboratory model}

For the laboratory in vivo model, six adult male beagle dogs approximately 1.5 years old were acquired after the approval of the Ethics Committee for Animal Research at Ecole National Veterinaire D'Alfort. 
Prior to general anesthesia, intramuscular atropine sulfate $(0.044 \mathrm{mg} / \mathrm{kg})$ and xylazine chlorate $(8 \mathrm{mg} / \mathrm{kg})$ were administered. A $15 \mathrm{mg} / \mathrm{kg}$ ketamine chlorate dose was then used to achieve general anesthesia.

The central region of the radius diaphysis was used for the surgery. After hair shaving, skin exposure, and antiseptic cleaning with iodine solution at the surgical and surrounding area, approximately a $5 \mathrm{~cm}$ length incision to access the periosteum was performed and a flap was thereafter reflected for bone exposure.

Four implants were placed along the radius from proximal to distal in an alternated distribution, with starting surface low $(\mathrm{L})$, medium $(\mathrm{M})$, high $(\mathrm{H})$, and control $(\mathrm{C})$ interchanged in every radius to minimize bias from the different implantation sites (sites 1 to 4 from proximal to distal). Therefore, the 48 implants remained in vivo for either one or six weeks (right and left radius provided samples that remained in vivo for one and six weeks, respectively), were allocated to sites one to four in an equal distribution. This approach resulted in balanced surgical procedures that allowed the comparison of the same number of implant surfaces per time in vivo, limb, surgical site (1 through 4$)$, and animal. The implants were placed at distances of $1 \mathrm{~cm}$ from each other along the central region of the bone.

After insertion, each implant received its proprietary cover screw to avoid tissue overgrowth. The soft tissue was sutured in layers according to standard procedures, with the periosteum and muscle layers sutured with Vicryl 4-0 (Ethicon, Johnson \& Johnson, Miami, FL) and the skin with 4-0 nylon (Ethicon).

Postoperative antibiotic and anti-inflammatory medications included a single dose of benzyl penicillin benzatine $(20,000 \mathrm{UI} / \mathrm{kg})$ intramuscularly and ketoprofen $1 \%(1 \mathrm{ml} / 5 \mathrm{~kg})$. The dogs were euthanized by an anesthesia overdose, and the limbs were retrieved by sharp dissection. The soft tissue was carefully removed by surgical blade, and an initial clinical evaluation was performed to determine implant stability. If an implant was clinically unstable, it was excluded from the study.

\section{Histologic procedures}

The retrieved implant-bone blocks were immersed in $10 \%$ buffered formalin solution for $24 \mathrm{~h}$, and afterwards were washed in running water for $24 \mathrm{~h}$. The fixated samples were then dehydrated in a series of alcohol solutions ranging from 70 to $100 \%$ ethanol. Following dehydration, the samples were embedded in a methacrylate-based resin (Technovit 9100, Heraeus Kulzer GmbH, Wehrheim, Germany) according to the manufacturer's instructions. The blocks were then cut into slices (approximately $300 \mu \mathrm{m}$ thickness) aiming the center of the implant along its long axis with a precision diamond saw (Isomet 2000, Buehler Ltd., Lake Bluff, USA), glued to acrylic plates with an acrylate-based cement, and a $24 \mathrm{~h}$ setting time was allowed prior to grinding and polishing. The sections were then reduced to a final thickness of approximately $30 \mu \mathrm{m}$ by means of a series of $\mathrm{SiC}$ abrasive papers (400, 600, 800, 1200 and 2400) (Buehler Ltd., Lake Bluff, IL, USA) in a grinding/polishing machine (Metaserv 3000, Buehler Ltd., Lake Bluff, IL, USA) under water irrigation. ${ }^{27}$ The sections were then stained with Stevenel's blue and van Gieson staining.

The BIC was determined at $50 \times-200 \times$ magnification (Leica DM2500M, Leica Microsystems GmbH, Wetzlar, Germany) by means of a computer software (Leica Application Suite, Leica Microsystems GmbH, Wetzlar, Germany). The regions of BIC along the implant perimeter were subtracted from the total implant perimeter, and calculations were performed to determine the BIC. The bone area fraction occupied (BAFO) between threads in trabecular bone regions was determined at $100 \times$ magnification (Leica DM2500M, Leica Microsystems GmbH, Wetzlar, Germany) by means of computer software (Leica Application Suite, Leica Microsystems GmbH, Wetzlar, Germany). The areas occupied by bone were subtracted from the total area between threads, and calculations were performed to determine the BAFO (reported in percentage values of bone area fraction occupied). ${ }^{28}$

IFM parameter statistical analysis was performed by one-way ANOVA and a mixed-model ANOVA was utilized for the in vivo evaluations (statistical unit considered the number of animal subjects, $\mathrm{n}=6$ ) considering implant surface and time in vivo as independent variables and BIC and BAFO as dependent variables. All analyses were conducted at $\alpha=0.05$.

\section{Results}

\section{Morphologic and topographic analysis}

SEM micrographs of all implant surfaces are presented in Figure 1 along with their representative $250 \times 250 \mu \mathrm{m}$ IFM three-dimensional reconstructions. Their respective micrometer length scale parameters $\mathrm{Sa}, \mathrm{Sq}$ and $\mathrm{Sdr}$ values are presented in Table 1.

The surface texture observed at low and high magnification levels of $\mathrm{C}, \mathrm{L}, \mathrm{M}$ and $\mathrm{H}$ roughness surface and the IFM reconstruction revealed morphologic differences among groups (Figure 1). The control surface presented qualitatively different surface patterning relative to all microrobot blasted surfaces, which presented higher degrees of uniformity. Qualitative analysis of residual alumina embedded in the textured surface showed that such condition was seldom observed 


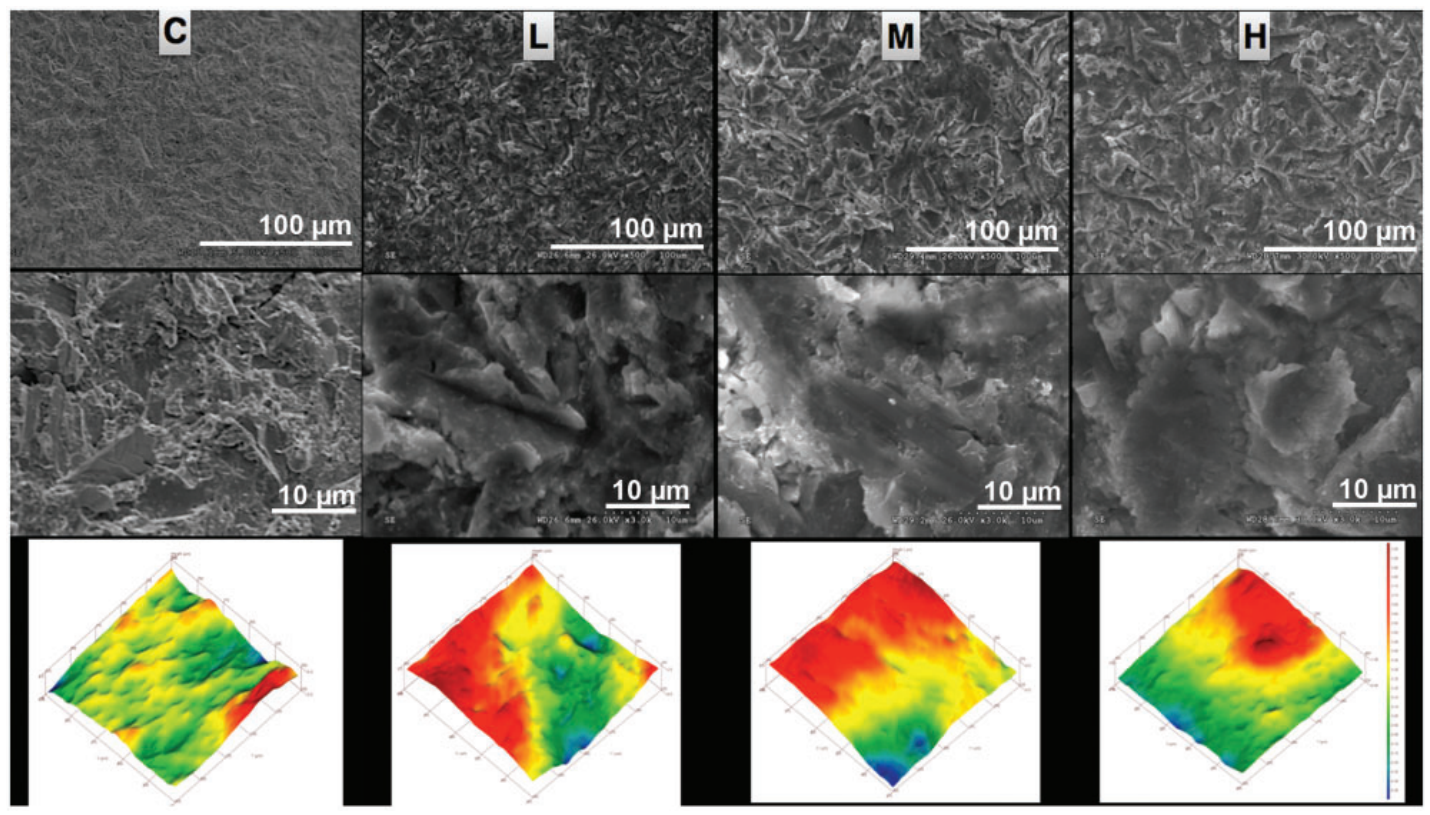

Figure I. SEM micrographs showing morphology of control (C), low (L), medium (M), and high surfaces $(H)$ at both low (top row) and high magnifications (middle row). IFM three-dimensional reconstruction is presented in the bottom row with a representative $125 \mu \mathrm{m} \times 125 \mu \mathrm{m}$ (scale presented in increments of $25 \mu \mathrm{m}$ ) region of interest, and each color scale increment represents $0.1 \mu \mathrm{m}$ (from $-1 \mu \mathrm{m}$ in blue to $3 \mu \mathrm{m}$ in red).

Table I. Interferometry roughness summary for the different implant groups reported as mean (SD).

\begin{tabular}{lllr}
\hline & Sa & Sq & \multicolumn{1}{l}{ Sdr } \\
\hline $\mathrm{H}$ & $1.43 \pm 0.07^{\mathrm{a}}$ & $1.77 \pm 0.15^{\mathrm{a}}$ & $90.3 \pm 12.3^{\mathrm{a}}$ \\
$\mathrm{M}$ & $1.31 \pm 0.08^{\mathrm{b}}$ & $1.65 \pm 0.14^{\mathrm{a}}$ & $67.5 \pm 10.6^{\mathrm{b}}$ \\
$\mathrm{L}$ & $0.67 \pm 0.07^{\mathrm{d}}$ & $0.84 \pm 0.14^{\mathrm{c}}$ & $50.1 \pm 9.6^{\mathrm{c}}$ \\
$\mathrm{C}$ & $0.87 \pm 0.18^{\mathrm{c}}$ & $1.18 \pm 0.2^{\mathrm{b}}$ & $47.16 \pm 9.5^{\mathrm{c}}$ \\
\hline
\end{tabular}

Note: Same superscript letters depict statistically homogeneous groups.

irrespective of experimental group. Although more uniform surface texturing patterns were observed for all microrobot blasted surfaces relative to the control, higher magnification images depicted differences in surface roughness pattern between the surfaces with a proportional increase in texture from low to high when compared to surface measured parameters (Figure 1). IFM measurements (Table 1) showed decreasing values of $\mathrm{Sa}, \mathrm{Sq}$ and $\mathrm{Sdr}$ values from the $\mathrm{H}$ to $\mathrm{L}$ surfaces. Control surfaces presented roughness parameter values higher or comparable to the $\mathrm{L}$ surface (Table 1).

\section{Histologic evaluation}

The animal surgical and postoperative procedures yielded no complications, infections or clinical concerns. No implants were excluded throughout the study due to clinical matters. A qualitative evaluation of the histological sections at one week showed that a healing chamber developed due to the implant and surgical instrumentation geometric interplay, where the inner part of the implant presented lower diameter than the outer diameter of the surgical instrumentation (Figure 2(a) to (d)). Overall, direct contact between pristine bone and the outer diameter part of the implant threads were observed for all groups. At one week, a fibrous connective tissue was presented at healing chamber regions of all different groups (Figure 2(a) to (d)). However, this connective tissue only presented osteogenic characteristics for the $\mathrm{H}$ surface group, where initial intramembranous-like formation was observed in direct contact with the implant surface and within the healing chamber volume (Figure 2(c)).

At six weeks, all surfaces presented extensive bone formation within the healing chambers. The bone within all groups' healing chambers presented bone formation in close proximity with the implant surfaces, within the healing chamber volume, and in direct contact with drilled pristine bone sites (Figure 2(e) to (h)). Primary osteonic strucutres were depicted around all samples (Figure 2(e) to (h)).

\section{Histomorphometric analysis}

Figure 3(a) shows the statistical summary for BIC when both times in vivo are collapsed. Significantly higher 


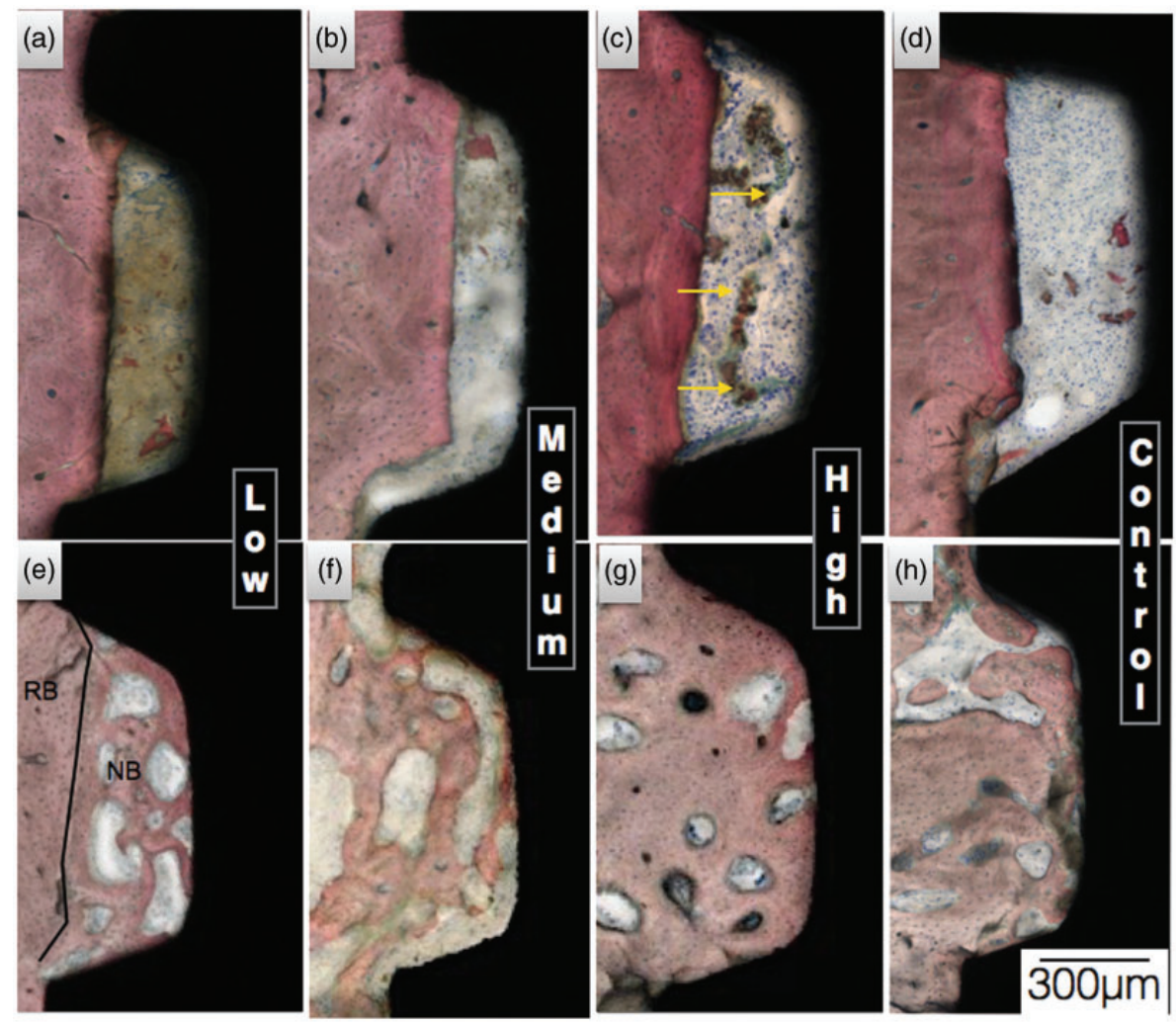

Figure 2. Histological section of the implants surface (a) low, (b) medium, (c) high, and (d) control at one week (a to d, respectively) and at six weeks ((e) to (h), respectively) in a beagle model. (c) Osteoblast line indicating the bone formation in close contact with the surface (yellow arrows). (e) Interface between the remodelling bone stages (RB) and new bone (NB). Stevenel's blue Von Giesson's staining.

BIC values were observed between the $H$ relative to $M$ and control samples. The L surface presented lower values than $\mathrm{H}$ (non-significant). When BIC was considered as a function of time in vivo, an overall significant increase was depicted between one and six weeks when all groups were collapsed or individually evaluated $(p<0.001)$ (Figure 3(b)).

At one week in vivo, the $\mathrm{H}$ and $\mathrm{L}$ groups presented significantly higher BIC values than the $\mathrm{M}$ surface, where only the $\mathrm{H}$ surface presented significantly higher values than control (Figure 3(b)). At six weeks, the $\mathrm{H}$ surface presented significantly higher values than $\mathrm{L}$ and Control groups (M groups presented intermediate values between $\mathrm{H}$ and $\mathrm{L}$, non-significant).

When BAFO was evaluated for each surface under collapsed time in vivo, a trend similar to BIC was observed where $\mathrm{H}$ presented significantly higher BAFO values than $\mathrm{M}$ and Control (L group presented intermediate values) (Figure 4(a)). At one week, the $\mathrm{H}$ group presented significantly higher BAFO values relative to all other groups. At six weeks, the $\mathrm{H}$ group presented significantly higher BAFO values relative to the $\mathrm{M}$ and control groups (L group presented intermediate values) (Figure 4(b)).

\section{Discussion}

Surface topography in the micrometer level is one of the decisive factors for the achievement of osseointegration. ${ }^{29}$ However, most of the micro roughening procedures that are applied to the commercial available implants of today are too broad, and to date, the optimal surface microtopography for improving osseointegration has not been confirmed. In an effort to identify the surface microtopography that would present the strongest bone responses, Wennerberg and Albrektsson have scrutinized the available in vivo animal studies and suggested that surface microroughness in a certain range presents the strongest bone responses. ${ }^{30}$ The surface roughness measurements in the current study presented that surfaces $\mathrm{H}$ and $\mathrm{M}$ were within the so-called moderately rough surface roughness in terms of the arithmetic average height deviation. The L surface and the control (C) surface presented significantly lower $\mathrm{Sa}$ as compared to the other 2 groups and the values were considered as minimally rough surfaces, which have suggested to present inferior osseointegration. ${ }^{31,32}$ The biological response confirmed in terms of BIC and BAFO presented were in general accordance with this tendency that surfaces 

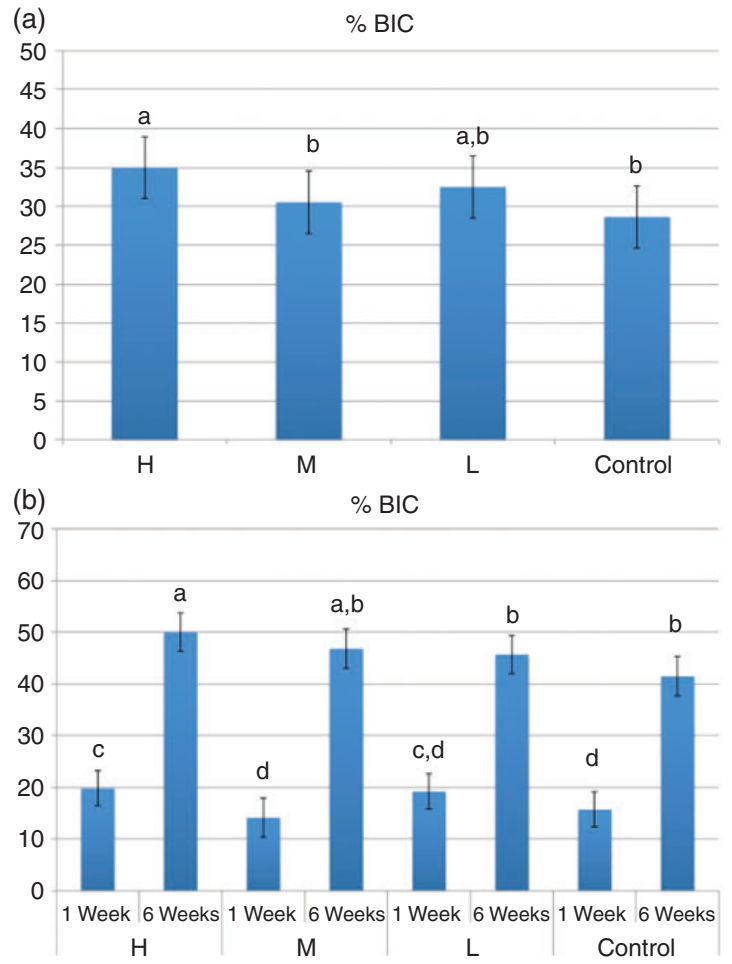

Figure 3. Box plot showing statistical summary (mean $\pm 95 \%$ $\mathrm{Cl}$ ) for \%BIC of the $\mathrm{L}, \mathrm{M}, \mathrm{H}$, and $\mathrm{C}$ surfaces (a), and \%BIC at one and six weeks in vivo (b). The asterisks depict statistically homogeneous groups (different letters depict that groups were statistically different at $p<0.05$ ).

$\mathrm{H}$ and $\mathrm{M}$ presented higher osseointegration than surface C. Moreover, the histological evaluation showed that surface $\mathrm{H}$ presented earlier onset of intramembranous-like formation in direct contact to the implant surface, which is an indication that surface $\mathrm{H}$ promotes early osteogenic activities.

It is notable that although the Sa value could be classified as minimally rough for the $\mathrm{L}$ surface, at certain times the early bone healing responses were comparable to that of surfaces $\mathrm{H}$ and $\mathrm{M}$. This is an indication that the microrobotized surface roughening procedure, which can control the blasting media size, velocity, and surface coverage, may generate a minimally rough surface from an arithmetic average height deviation perspective, however, could achieve high levels of osseointegration. It is also an indication that the proposed surface microtexturing procedure has the possibility to selectively alter different surface topographical parameters, in this case, not increasing the height parameter.

As it happens, the recent trend in implant surface modification is to lower the surface roughness, in terms of Sa value, however, at the same time, promote comparable osseointegration to the so-called moderately rough implant surfaces. ${ }^{33}$ It has been reported
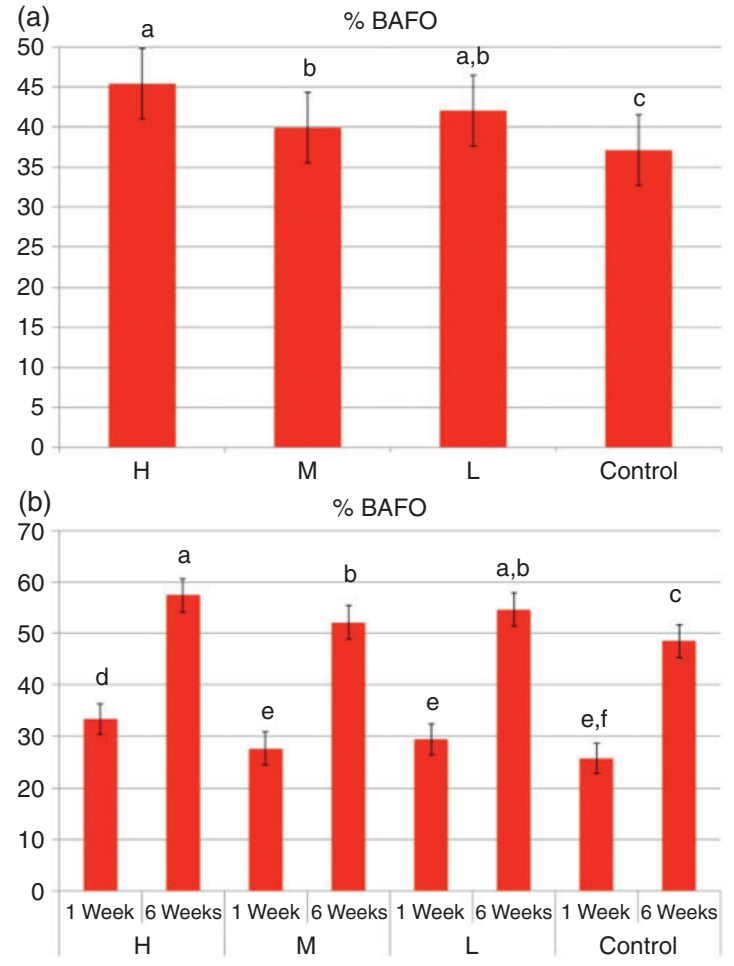

Figure 4. Box plot showing statistical summary (mean $\pm 95 \%$ $\mathrm{Cl}$ ) for \%BAFO of the L, M, H and C surfaces (a) and \%BAFO at one and six weeks in vivo (b). The letters depict statistically homogeneous groups (different letters depict that groups were statistically different at $p<0.05$ ).

by Halldin et al. that dual acid-etched implants with a reduced microtopography in height deviation, presented equivalent osseointegration compared to the commercially available fluoride-treated implants in a biomechanical investigation using rabbits. ${ }^{34}$ Thus, it can be speculated that parameters other than the arithmetic average height deviation was altered in a manner that it stimulated osteogenesis for surface L.

Surface roughness consists of numerous parameters such as amplitude (height), spatial, hybrid and even functional and each parameter theoretically influence the biological responses. ${ }^{35}$

In the present study, the blasting variables were regulated during the microrobotized blasting procedure, which resulted in three different surfaces with unique surface topographies. It can be suggested that many of the surface topographical parameters were altered during this process. With the optical light interferometer, the amplitude and hybrid parameters of these surfaces were evaluated and the biologic responses to the different surfaces somewhat varied. However, it should be noted that in the majority of the implant surface modification studies available in the literature, the amplitude parameter was the central focus and the effect of other parameters was seldom discussed. 
A limitation of the present study is the lack of a thorough surface chemistry characterization between groups given as in vitro studies have pointed that different levels of alumina embedding in the implant surface may change cellular events in osteogenic cell lines. ${ }^{36,37}$ While our electron microscopy evaluation did not show overt qualitative differences in alumina embedding between surfaces, the differences in processing may be quantitatively different and such quantification may be used as an ossoeintegration likelihood indicator and thereby should be considered in future investigations. The results of the current in vivo study, however, suggest that there may be other parameters that influence osseointegration and may be of significant interest in the future to determine whether other parameters can alter the degree of amplitude. With the microrobotized blasting procedure, altering the different parameters may be conducted in a controlled manner and may shed light to improved surface topography that present stronger bone responses.

\section{Declaration of Conflicting Interests}

The author(s) declared no potential conflicts of interest with respect to the research, authorship, and/or publication of this article.

\section{Funding}

The author(s) disclosed receipt of the following financial support for the research, authorship, and/or publication of this article: To Conselho Nacional de Desenvolvimento Científico e Tecnológico (CNPq), grant \# 309475/2014-7.

\section{References}

1. Henry PJ. Implant hardware - science or commodity development? J Dent Res 1995; 74: 301-302.

2. Albrektsson T, Branemark PI, Hansson HA, et al. Osseointegrated titanium implants. Requirements for ensuring a long-lasting, direct bone-to-implant anchorage in man. Acta Orthop Scand 1981; 52: 155-170.

3. Elias CN, Rocha FA, Nascimento AL, et al. Influence of implant shape, surface morphology, surgical technique and bone quality on the primary stability of dental implants. J Mech Behav Biomed Mater 2012; 16: 169-180.

4. Jimbo R, Tovar N, Anchieta RB, et al. The combined effects of undersized drilling and implant macrogeometry on bone healing around dental implants: an experimental study. Int J Oral Maxillofac Surg 2014; 43: 1269-1275.

5. Coelho PG, Granato R, Marin C, et al. The effect of different implant macrogeometries and surface treatment in early biomechanical fixation: an experimental study in dogs. J Mech Behav Biomed Mater 2011; 4: 1974-1981.

6. Dos Santos MV, Elias CN and Lima CJH. The effects of superficial roughness and design on the primary stability of dental implants. Clin Implant Dent Rel Res 2011; 13: 215-223.
7. Jimbo R, Janal MN, Marin C, et al. The effect of implant diameter on osseointegration utilizing simplified drilling protocols. Clin Oral Implants Res 2014; 25: 1295-1300.

8. Coelho PG and Jimbo R. Osseointegration of metallic devices: current trends based on implant hardware design. Arch Biochem Biophys 2014; 561: 99-108.

9. Coelho PG, Bonfante EA, Pessoa RS, et al. Characterization of five different implant surfaces and their effect on osseointegration: a study in dogs. J Periodontol 2011; 82: 742-750.

10. Coelho PG, Takayama T, Yoo D, et al. Nanometer-scale features on micrometer-scale surface texturing: a bone histological, gene expression, and nanomechanical study. Bone 2014; 65: 25-32.

11. Teixeira HS, Marin C, Witek L, et al. Assessment of a chair-side argon-based non-thermal plasma treatment on the surface characteristics and integration of dental implants with textured surfaces. J Mech Behav Biomed Mater 2012; 9: 45-49.

12. Coelho PG, Jimbo R, Tovar N, et al. Osseointegration: Hierarchical designing encompassing the macrometer, micrometer, and nanometer length scales. Dent Mater 2015; 31: 37-52.

13. Buser D, Schenk RK, Steinemann S, et al. Influence of surface characteristics on bone integration of titanium implants. a histomorphometric study in miniature pigs. J Biomed Mater Res 1991; 25: 889-902.

14. Wennerberg A, Albrektsson $\mathrm{T}$ and Andersson B. An animal study of c.p. titanium screws with different surface topographies. J Mater Sci Mater Med 1995; 6: 302-309.

15. Sul YT, Johansson C and Albrektsson T. Which surface properties enhance bone response to implants? Comparison of oxidized magnesium, TiUnite, and Osseotite implant surfaces. Int J Prosthodont 2006; 19: 319-328.

16. Kang B-S, Sul Y-T, Oh S-J, et al. XPS, AES and SEM analysis of recent dental implants. Acta Biomater 2009; 5: 2222-2229.

17. Lemons JE. Biomaterials, biomechanics, tissue healing, and immediate-function dental implants. $J$ Oral Implantol 2004; 30: 318-324.

18. Coelho PG, Granjeiro JM, Romanos GE, et al. Basic research methods and current trends of dental implant surfaces. J Biomed Mater Res B Appl Biomater 2009; 88: 579-596.

19. Albrektsson T and Wennerberg A. Oral implant surfaces: Part 1 - review focusing on topographic and chemical properties of different surfaces and in vivo responses to them. Int J Prosthodont 2004; 17: 536-543.

20. Wennerberg A and Albrektsson T. On implant surfaces: a review of current knowledge and opinions. Int $J$ Oral Maxillofac Implants 2010; 25: 63-74.

21. Berglundh T, Abrahamsson I, Lang NP, et al. De novo alveolar bone formation adjacent to endosseous implants. Clin Oral Implants Res 2003; 14: 251-262.

22. Jimbo R, Sawase T, Shibata Y, et al. Enhanced osseointegration by the chemotactic activity of plasma 
fibronectin for cellular fibronectin positive cells. Biomaterials 2007; 28: 3469-3477.

23. Zhang L and Webster TJ. Nanotechnology and nanomaterials: promises for improved tissue regeneration. Nano Today 2009; 4: 66-80.

24. Xia L, Lin K, Jiang X, et al. Enhanced osteogenesis through nano-structured surface design of macroporous hydroxyapatite bioceramic scaffolds via activation of ERK and p38 MAPK signaling pathways. $J$ Mater Chem B 2013; 1: 5403.

25. Johansson $\mathrm{CB}$, Gretzer C, Jimbo R, et al. Enhanced implant integration with hierarchically structured implants: a pilot study in rabbits. Clin Oral Implants Res 2012; 23: 943-953.

26. Valverde GB, Jimbo R, Teixeira HS, et al. Evaluation of surface roughness as a function of multiple blasting processing variables. Clin Oral Implants Res 2013; 24: 238-242.

27. Donath K and Breuner G. A method for the study of undecalcified bones and teeth with attached soft tissues. The Sage-Schliff (sawing and grinding) technique. J Oral Pathol 1982; 11: 318-326.

28. Leonard G, Coelho P, Polyzois I, et al. A study of the bone healing kinetics of plateau versus screw root design titanium dental implants. Clin Oral Implants Res 2009; 20: 232-239.

29. Wennerberg A, Albrektsson $\mathrm{T}$ and Jimbo R. Implant surfaces and their biological and clinical impact. Heidelberg, Germany: Springer-Verlag Berlin Heidelberg, 2015.
30. Wennerberg A and Albrektsson T. Effects of titanium surface topography on bone integration: a systematic review. Clin Oral Implants Res 2009; 20: $172-184$.

31. Wennerberg A, Ide-Ektessabi A, Hatkamata S, et al. Titanium release from implants prepared with different surface roughness. An in vitro and in vivo study. Clin Oral Implants Res 2004; 15: 505-512.

32. Albrektsson T, Sennerby L and Wennerberg A. State of the art of oral implants. Periodontology 2000 2008; 47: 15-26.

33. Simion M, Benigni M, Al-Hezaimi K, et al. Early bone formation adjacent to oxidized and machined implant surfaces: a histologic study. Int $J$ Periodont Rest Dent 2015; 35: 9-17.

34. Halldin A, Jimbo R, Johansson CB, et al. Improved osseointegration and interlocking capacity with dual acid-treated implants: a rabbit study. Clin Oral Implants Res 2014; 27: 22-30. DOI: 10.1111/clr.12507.

35. Wennerberg A and Albrektsson T. Suggested guidelines for the topographic evaluation of implant surfaces. Int $J$ Oral Maxillofac Implants 2000; 15: 331-344.

36. Canabarro A, Diniz MG, Paciornik S, et al. High concentration of residual aluminum oxide on titanium surface inhibits extracellular matrix mineralization. $J$ Biomed Mater Res A 2008; 87: 588-597.

37. Sader MS, Balduino A, Soares Gde A, et al. Effect of three distinct treatments of titanium surface on osteoblast attachment, proliferation, and differentiation. Clin Oral Implants Res 2005; 16: 667-675. 